\title{
Bedload Transport Quantification using Image Processing Techniques
}

\author{
Alexander Anatol ERMILOV ${ }^{1, \bowtie}$, Slaven CONEVSKI ${ }^{2, \bowtie}$, \\ Massimo GUERRERO ${ }^{3, \bigotimes}$, Sandor BARANYA ${ }^{1}$, Nils RUTHER ${ }^{2}$, and Gabor FLEIT ${ }^{1}$ \\ ${ }^{1}$ Department of Hydraulic and Water Resources Engineering, \\ Budapest University of Technology and Economics, Budapest, Hungary \\ ${ }^{2}$ Department of Civil and Environmental Engineering, \\ Norwegian University of Science and Technology, Norway, Trondheim \\ ${ }^{3}$ Department of Civil, Chemical, Environmental, and Materials Engineering, University of Bologna, \\ Bologna, Italy \\ $\triangle$ ermilov.alexander@emk.bme.hu; slaven.conevski@ntnu.no; massimo.guerrero@unibo.it
}

\begin{abstract}
Image processing techniques we deployed to measure bedload transport characteristics. The bedload velocity was successfully measured and in some cases the bedload concentration and particle size were estimated. Two different approaches were used and good corelations were reported from both the laboratory and the field data. Comparing the field video results to conventional bedload sampling measurements showed promising results for further development of the image-based techniques. Moreover, the captured videos could help understanding the performance of the conventional samplers, pointing out typical sampling errors.
\end{abstract}

Keywords: sediment transport, image processing, bedload, image velocimetry, bedload monitoring.

\section{INTRODUCTION}

In the last few decades, various videography techniques have been developed to investigate the behavior of the bedload particles, mostly on a small scale and in controlled conditions. These techniques are usually applied for very weak transports and mostly to examine the incipient motion of the particles. The image processing techniques such as optical flow and image differencing have been successfully applied to calculate the mobile bed velocity, the surface concentration of mobile particles and the shape of the particles when gravel transport takes place, both in laboratory and field environment (Radice et al. 2006; Conevski et al. 2019; Ermilov et 
al. 2020). In this study, two image-based methods are implemented and their applicability in various bedload transport conditions is discussed.

\section{METHODOLOGY AND RESULT}

\subsection{Experimental set ups and data post- processing}

GoPro cameras (4k, $2.7 \mathrm{k}$ and 1080 p; 30 and $60 \mathrm{fps}$ ) were used to record videos for both laboratory and field measurements. In the laboratory the camera was mounted at the centre of the flume filled with sediment material which had a bedload trap at the end measuring the bedload mass at $1 \mathrm{~Hz}$. Three different bedload transport conditions were performed with medium sand $(0.35 \mathrm{~mm})$ and fine gravel $(7 \mathrm{~mm})$. In the latter case, the device was fixed to a bedload sampler and was lowered from a boat to the riverbed. Underwater lights ensured adequate light source and visibility in both experiments.

The image data processing followed the same procedure as explained in Conevski et al. (2019), involving image enhancement, image de-blurring and applying the image subtraction method (i.e., two-frame change detection). The excluded changes are considered as single particles (e.g., gravel) or fluxes of particles movements.

The second method, applied for the field data, was based on the statistical background method extraction. The method is adaptive, e.g., when a particle settles the background will be updated. Then, the same procedure of subtracting and identifying the background and foreground pixels is applied in both methods. The false pixels were filtered out by using threshold and low pass filtering. Next, the velocities are calculated by using cross correlation methods (i.e., PIV methodology). A cut off velocities derived from the measured water velocities were used to screen the final velocity outputs. The particle size (e.g., gravel bed) and the bedload concentration (e.g., sandy bed and sand-gravel) were calculated based on the detected foreground pixels.

\subsection{Results and discussion}

Good correlation coefficients (Pearson), between bedload transport rate and the camera measured velocities, were observed in both lab $(r \sim 0.8$, Fig. 1a) and field study $(r \sim 0.6)$. The measurement with sandy sediments gave higher deviation due to the random resuspension of particles from the bedload. By considering a characteristic sediment density and converting the particle size from pixel to millimetres, the mass of the bedload transport was estimated (Fig. 1b).

a)

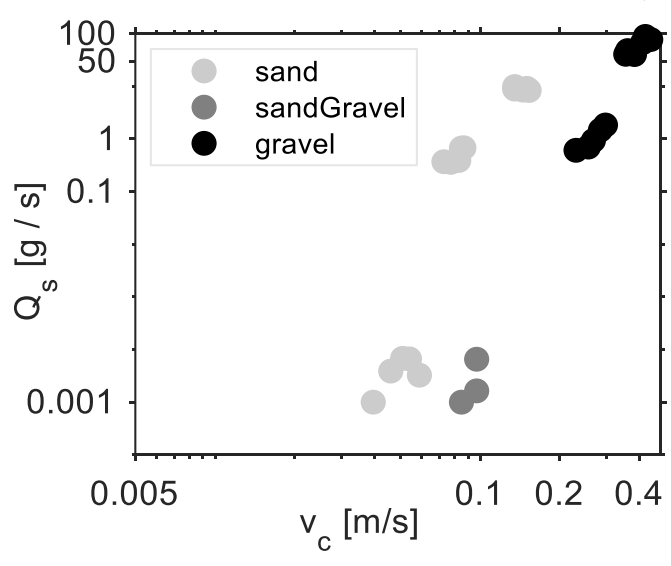

b)

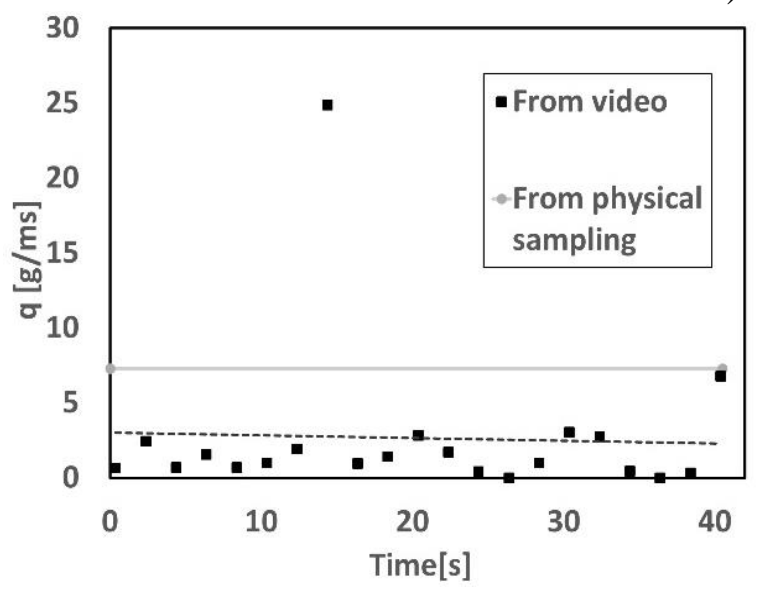

Fig. 1: a) Camera velocities vs bedload transport rate (lab), b) field data - calculated mass of bedload per unit width (field). 
The different methods and their results highlighted the possible limitations (e.g. high suspended sediment concentration - poor visibility). Nevertheless, at lower sediment transport conditions, image-based techniques are for a good alternative and can significantly decrease the time- and labor demand of bedload sampling measurement campaigns.

\section{References}

Conevski, S., M. Guerrero, N. Ruther, and C.D. Rennie (2019), Laboratory investigation of apparent bedload velocity measured by ADCPs, J. Hydraul. Eng. 145, 11, DOI: 10.1061/(ASCE)HY. 1943-7900.0001632.

Radice, A., S. Malavasi, and F. Ballio (2006), Solid transport measurements through image processing, Exp. Fluids 41, 5, 721-734, DOI: 10.1007/s00348-006-0195-9.

Ermilov, A.A., S. Baranya, G. Fleit, and G.T. Török (2020), Developing image-based methods for analysing morphodynamics in large rivers, Hidrológiai Közlöny 100, 3, 74-86.

Received 22 March 2021

Accepted 12 April 2021 